\title{
Entre égalité de traitement et harcèlement, quel fondement juridique de la discrimination syndicale?
}

État des lieux et analyse du contentieux entre 2012 et 2014

What Legal Foundations for Anti-Union Discrimination in France? Inventory and Analysis of Litigations Between 2012 and 2014

Frédéric Guiomard et Inès Meftah

\section{revues.org}

Édition électronique

URL : http://travailemploi.revues.org/6895

ISSN : 1775-416X

\section{Éditeur}

DARES - Ministère du travail de la solidarité et de la fonction publique

\section{Édition imprimée}

Date de publication : 1 janvier 2016

Pagination : 59-85

ISSN : 0224-4365

Distribution électronique Cairn

\section{CAIRN INFO}

CHERCHER, REPÉRER, AVANCER.

Référence électronique

Frédéric Guiomard et Inès Meftah, «Entre égalité de traitement et harcèlement, quel fondement juridique de la discrimination syndicale? », Travail et Emploi [En ligne], 145 I janvier-mars 2016, mis en ligne le 01 janvier 2018, consulté le 04 novembre 2016. URL : http://travailemploi.revues.org/6895 ; DOI : 10.4000/travailemploi.6895

Ce document est un fac-similé de l'édition imprimée.

(c) La documentation française 


\title{
Entre égalité de traitement et harcèlement, quel fondement juridique de la discrimination syndicale ?
}

\author{
État des lieux et analyse du contentieux entre 2012 et 2014
}

\author{
Frédéric Guiomard ${ }^{*}$, Inès Meftah ${ }^{* *}$
}

\begin{abstract}
Le droit français connaît depuis 1956, sous l'influence du droit international et européen, le développement d'un important arsenal législatif destiné à lutter contre les discriminations fondées sur l'appartenance ou l'activité syndicale. Si ces normes et leur interprétation juridique sont bien connues, les contentieux qu'elles génèrent ne font l'objet d'aucune évaluation. La présente recherche permet de dresser un état des lieux de ces contentieux, éclatés devant différents ordres de juridictions, et d'en comprendre les principales caractéristiques. L'analyse révèle un contentieux relativement peu fourni, portant sur des questions variées, ainsi que la relative imprécision de ce fondement de l'action en justice, qui parvient rarement à s'émanciper d'autres questions telles que l'égalité de traitement ou le harcèlement, y perdant ainsi une partie de sa spécificité. Il semble en conséquence que les normes anti-discriminatoires, par distinction des autres règles garantissant l'exercice des droits syndicaux, soient mobilisées essentiellement pour garantir le respect de droits individuels, par opposition à la garantie de prérogatives d'action collective.
\end{abstract}

T es discriminations syndicales sont aussi anciennes que le syndicalisme et Ldemeurent répandues malgré le développement d'un arsenal juridique conséquent (VERDIER, 1987) (voir encadré). Leur caractère dissimulé (les employeurs demeurant le plus souvent prudents en raison du risque de sanctions pénales) rend néanmoins très incertaine l'observation de ces pratiques et de la manière dont les règles juridiques sont utilisées pour les combattre (CHAPPE, 2013). Pour le juriste, l'analyse des contentieux qu'elles suscitent est donc un outil privilégié de la compréhension de ces normes, de leur invocation par les justiciables et de leur application par les juges (GUIOMARD, MEFTAH, 2013). Elle permet de révéler la diversité des pratiques syndicales, des formes

\footnotetext{
* Université Paris Ouest Nanterre La Défense. Institut de recherche sur l'entreprise et les relations professionnelles (Irerp) ; frederic.guiomard@u-paris10.fr.

** Université Paris Ouest Nanterre La Défense, Irerp ; ines.meftah@ hotmail.fr.
} 
prises par les discriminations, ainsi que de la mobilisation des règles juridiques à l'encontre de ces pratiques (SPIRE, 2006).

Historiquement, la protection contre les discriminations a connu une évolution importante depuis une trentaine d'années. La première génération de droits, promue à la suite de l'adoption de la loi du 27 avril 1956, visait avant tout à protéger l'exercice d'une liberté publique dans l'entreprise (LANQueTIN, 2010). Comme l'indique JeanMaurice VERDIER (1984, p. 140), « elle tend à protéger l'action syndicale, fondée certes sur l'activité militante individuelle, mais en elle-même essentiellement collective ». Cette protection concerne par conséquent avant tout les prérogatives syndicales, reposant sur un statut protecteur accordé aux représentants du personnel à partir de l'ordonnance du 22 février 1945, qui exige une autorisation administrative pour leur licenciement (voir encadré).

Une nouvelle génération de droits va éclore à compter de la loi du 4 août 1982. En incluant la prohibition des licenciements et sanctions fondées sur les activités syndicales dans la liste des discriminations prohibées, le législateur français a fait le choix d'inscrire ces règles dans le giron des droits de la personne, permettant d'enrichir la lutte contre les discriminations. Ces dispositions furent complétées par l'adjonction des discriminations syndicales dans la liste des discriminations pénalement sanctionnées lors de l'adoption du Code pénal de $1994^{1}$, et par l'adoption d'un régime spécifique pour la définition, l'action en justice et la preuve des discriminations ${ }^{2}$.

De façon générale, la discrimination se définit comme la prohibition des actes désavantageant une personne pour un motif prohibé par la loi. Sous l'influence de l'Union européenne, le droit français interdit non seulement les discriminations directes par lesquelles la personne est traitée de façon moins favorable en raison de l'un des motifs prohibés, mais aussi les discriminations indirectes par lesquelles une pratique apparemment neutre désavantage particulièrement les membres d'un groupe protégé contre les discriminations ${ }^{3}$.

\section{ENCADRÉ}

Le cadre juridique de la protection des représentants du personnel et des militants syndicaux

Trois corps de règles se combinent pour assurer l'exercice de la liberté syndicale et des droits syndicaux dans l'entreprise :

- les adhérents aux syndicats (auxquels peuvent être assimilés les simples sympathisants) se voient reconnaître le droit à la liberté syndicale (art. L. 2141-1 et suivants du Code du travail). Ils bénéficient en outre d'une protection contre les discriminations en matière notamment de recrutement, de licenciement, de rémunération ou de carrière, fondées sur

1. Article (art.) L. 225-1, Code pénal.

2. Art. L. 1134-1 et suivants, Code du travail.

3. Directive 2000/78/CE du 27 novembre 2000 portant création d'un cadre général en faveur de l'égalité de traitement en matière d'emploi et de travail, art. 2. 
Entre égalité de traitement et harcèlement, quel fondement juridique de la discrimination syndicale ?

les activités syndicales ou les opinions politiques (art. L. 1132-1 et L. 2141-5 du Code du travail), la mesure étant susceptible de nullité et/ou de réparation indemnitaire. Par ailleurs l'article 225-1 du Code pénal institue une sanction pénale pour les discriminations fondées sur les opinions politiques et les activités syndicales ;

- les représentants du personnel élus ou désignés bénéficient de leur côté d'un statut protecteur : il consiste en un ensemble de règles d'ordre public qui les protègent dans l'entreprise contre les pressions de l'employeur et leur garantissent l'indépendance dans l'exercice de leur mandat. Trois types de dispositions peuvent être alors distingués :

- des règles garantissant l'exercice des mandats : heures de délégation, libre circulation dans l'entreprise. En cas de non-respect, les manquements sont sanctionnés pour l'essentiel par la sanction pénale du délit d'entrave (art. L. 2146-1 du Code du travail),

- des règles protégeant le salarié contre la rupture de son contrat de travail : tout licenciement est soumis à une autorisation préalable de licenciement de l'inspection du travail (art. L. 2411-1 et suivants). La procédure est également applicable à la rupture des contrats de travail à durée déterminée (art. L. 2412-1), au transfert du contrat de travail (art. L. 2414-1) et à la rupture conventionnelle (art. L. 1237-15). La rupture du contrat de travail au mépris de ces règles est sanctionnée par le droit à réintégration des salariés (art. L. 2422-1), et/ou par des sanctions pénales (art. L. 2431-1),

- la jurisprudence a également permis le développement d'actions fondées sur les règles de non-discrimination syndicale en cas de traitement différencié subi par les représentants du personnel. Les différences peuvent porter sur la rémunération, la classification ou encore le déroulement de la carrière. Les contentieux qui en découlent, engagés depuis la fin des années 1990, aboutissent le plus souvent à l'utilisation de panels de comparaison permettant de mettre en regard la situation des représentants du personnel avec celle des autres salariés exerçant de façon comparable et recrutés au même moment. Ces actions permettent d'obtenir à la fois des rattrapages de salaire et des repositionnements dans les grilles de classification. Elles ont conduit les entreprises à développer des accords sur la carrière des représentants du personnel, devenue l'objet d'une négociation collective obligatoire avec la loi du 20 août 2008 (art. L. 2141-5, alinéa 2). Depuis la loi Rebsamen du 15 août 2015, une négociation doit s'engager au minimum tous les trois ans, dans les entreprises de plus de trois cents salariés, sur le déroulement de carrière des salariés exerçant des responsabilités syndicales et l'exercice de leurs fonctions (art. L. 2242-13 $6^{\mathrm{e}}$ du Code du travail);

- enfin, les militants syndicaux et représentants du personnel se voient appliquer l'ensemble des garanties offertes aux autres salariés contre l'exercice des prérogatives de l'employeur : protection contre le licenciement injustifié, droit disciplinaire, principe « à travail égal, salaire égal », application des règles de classification et des minima conventionnels, protection contre les atteintes aux droits des personnes et aux libertés individuelles et collectives de l'article L. 1221-1 du Code du travail, etc. Ces différentes règles permettent d'obtenir une protection contre les mesures de rétorsion et les inégalités de traitement subies sans qu'il soit nécessaire d'invoquer le motif discriminatoire animant l'employeur. 
Ces normes peuvent être mobilisées devant tous les ordres de juridictions : les juridictions administratives sont compétentes pour les litiges relatifs aux autorisations de licenciement des salariés protégés ${ }^{4}$; les juridictions du travail, en première instance les conseils des prud'hommes, pour les litiges consécutifs aux licenciements prononcés en méconnaissance d'une autorisation ou relatifs à la discrimination dans la carrière, également pour une sous-rémunération ou tout acte défavorable pris contre les représentants du personnel ${ }^{5}$; les juridictions civiles (les tribunaux d'instance ou de grande instance avant d'éventuels appels) pour les litiges d'ordre collectif entre syndicats et employeurs ; les juridictions répressives (comme le tribunal correctionnel) pour les litiges portant sur les discriminations en raison des activités syndicales ${ }^{6}$.

Comme nous allons le voir, l'analyse des décisions rendues montre la multitude des objets de litiges : licenciements au mépris de règles protectrices des titulaires de mandats, écarts de rémunération, retards de carrières, processus d'évaluation dévoyés, etc. Les décisions n'en révèlent pas moins des logiques patronales variées, selon qu'elles concernent des mesures prises à l'encontre de la présence syndicale dans l'entreprise, des pressions à l'égard des représentants du personnel dans le cadre d'un rapport de force inhérent aux relations professionnelles, de mesures à l'encontre des représentants d'une obédience syndicale particulière ou d'un militant.

Le prisme contentieux est particulièrement éclairant pour observer ces pratiques, dans la mesure où les tribunaux constituent « un lieu transversal de traitement des revendications », dont l' analyse permet de rendre compte des configurations litigieuses et stratégies procédurales déployées par les protagonistes (SERVERIN, GUIOMARD, 2013, p. 20). Les actions en justice ne sauraient en effet être comprises comme le produit d'une application automatique des normes à des hypothèses préalablement envisagées par le législateur. Elles sont le produit de l'appropriation par les plaideurs des dispositifs permettant de saisir la justice dans le but d'obtenir un résultat économique ou social escompté. Le choix de la juridiction devant laquelle le litige est porté, des procédures, des objets de litige et des arguments déployés sont ainsi à comprendre dans le contexte d'une rationalité stratégique plus ou moins maîtrisée par les plaideurs. Ceux-ci mobilisent devant les juridictions les ressources dont ils disposent - qu'elles soient matérielles (financement du coût de l'action, établissement des preuves) ou cognitives (connaissance des outils juridiques et processuels) - afin d'obtenir satisfaction. Dès lors, le prisme des contentieux peut paraître quelque peu déformant pour qui imaginerait le droit comme un processus statique de réalisation des normes dans un sens préalablement déterminé par le législateur. Si de nombreuses affaires illustrent l'utilisation de ces normes pour lutter contre des comportements anti-syndicaux, d'autres montrent la poursuite par les plaideurs de finalités plus diffuses telles que l'amélioration de la réparation au titre d'une action engagée sur un fondement principal extérieur aux discriminations ou l'instrumentalisation de l'action en justice dans le cadre de stratégies de négociation

4. Art. L. 2411-1 et suivants, Code du travail.

5. Art. L. 1132-1 et L. 2141-5, Code du travail.

6. Art. 225-1, Code pénal ; art. L. 2146-2, Code du travail. 
collective ou de conflit entre une organisation syndicale et un employeur. Il est en conséquence important de déceler ces stratégies de mobilisation des règles juridiques afin de comprendre les fonctions remplies par ces règles dans l'ordre juridique.

Il est à ce titre capital de préciser que l'analyse des contentieux ne rend compte que des actions en justice et ne permet donc l'observation directe ni des pratiques illicites qui ne sont pas contestées en justice, ni de celles qui, spontanément, permettent une application des règles de droit. La présence ou l'absence de contentieux ne peut donc pas être interprétée comme le signe d'un plus ou moins grand respect des règles de droit.

Dans la perspective d'étudier les décisions portant proprement sur les discriminations syndicales, le choix a été fait d'examiner les recours portés devant les différentes juridictions à travers un échantillon de décisions rendues par les juridictions d'appel (cours d'appel et cours administratives d'appel), dont les décisions sont aujourd'hui presque intégralement mises à disposition sur la base Lamyline, qui reprend le fonds Jurica de la Cour de cassation (SERVERIN, 2009). L'étude, ainsi présentée, complète les analyses plus générales menées sur les décisions rendues sur l'ensemble de la question des discriminations par les cours d'appel (Bossu, 2014 ; SERVERIN, GuiOMARD, 2013). Elle couvre l'ensemble des décisions rendues entre le $1^{\text {er }}$ janvier 2012 et le 31 décembre 2014. L'interrogation de la base de données a consisté à effectuer une recherche en plein texte de l'expression « discrimination syndicale ». La difficulté d'un tel travail provient de l'abondance des décisions à analyser. Le choix a donc été fait de travailler à la fois sur cet échantillon pour comprendre la structure de l'ensemble des contentieux, parfois complété par un examen des décisions de la Cour de cassation et du Conseil d'État pour affiner certaines données, ainsi que sur des études de sous-échantillons portant sur des périodes plus courtes permettant une analyse plus fine, sans avoir à travailler sur des masses trop considérables d'affaires. L'étude a alors couvert l'ensemble des décisions rendues au cours du dernier trimestre de 2014 par les cours d'appel et cours administratives d'appel'.

Le choix de cette méthodologie conduit à étudier exclusivement les litiges impliquant explicitement des questions de discrimination, que cela soit l'objet principal du litige ou d'arguments accessoires déployés. Les multiples règles par lesquelles les militants et représentants syndicaux peuvent demander la garantie de leurs droits d'agir dans l'entreprise (voir encadré) conduisent nécessairement à se fonder seulement sur les décisions dans lesquelles est ouvertement mobilisée cette problématique. Les stratégies de mobilisation du droit rendent indécelables les affaires, sans doute nombreuses, dans lesquelles les salariés agissent dans la perspective de lutter contre un comportement trouvant son origine dans une attitude discriminatoire - le plus souvent impossible à prouver - en déplaçant le litige sur un fondement assurant une meilleure chance d'obtenir satisfaction : principe d'égalité, liberté d'expression, sanction ou licenciement injustifié, etc. Le régime particulier des discriminations n'est utile aux plaideurs que lorsqu'ils réclament les sanctions les plus fermes (sanctions pénales, 
réintégration), mais non pour les réparations indemnitaires, qui peuvent être menées sur d'autres fondements. La présente étude ne permet en conséquence pas de mesurer quantitativement l'ensemble des contentieux qui renvoient, plus ou moins directement, à des comportements discriminatoires ; elle permet toutefois de saisir les principales formes que prennent les actions en justice qui les invoquent explicitement.

L'ambition de la présente étude est de montrer les principales caractéristiques du contentieux des discriminations syndicales. À cet effet, deux dimensions peuvent être mises en avant : la variété du rôle joué par ces normes, à travers la multiplicité des types de contentieux engagés, que nous détaillerons dans notre première partie ; et, sur un plan plus qualitatif, le caractère profondément ambivalent de la construction d'une partie de ces litiges, que nous analyserons dans la seconde.

\section{Une typologie des contentieux en matière de discrimination syndicale}

L'observation des contentieux relatifs aux discriminations syndicales permet de comprendre la fonction occupée par ces discriminations dans l'ordre juridique, à tout le moins la place que leur reconnaissent les différents acteurs lorsqu'ils mobilisent les règles du droit. À défaut de pouvoir proposer une classification exhaustive en raison de la multiplicité et de la complexité des litiges, il est possible d'en dégager les principales caractéristiques.

L'invocation des discriminations est avant tout portée par des salariés devant des juridictions civiles (voir tableau 1). Devant la Cour de cassation, un total de 253 décisions a été rendu sur ce thème, 236 étant portées devant la chambre sociale (litiges prud'homaux à l'origine), 15 devant la chambre criminelle (contentieux répressifs) et 2 devant la $2^{\mathrm{e}}$ chambre civile (spécialisée dans les questions de procédure civile et de sécurité sociale $)^{8}$. Les ordres de grandeur sont probablement comparables en appel, mais l'absence d'exhaustivité des bases de données les concernant rend impossible leur évaluation quantitative. En comparaison, les litiges administratifs sont peu nombreux : 41 décisions du Conseil d'État et 210 décisions de cours administratives d'appel se réfèrent à la discrimination syndicale ou au caractère discriminatoire des licenciements pour lesquels une autorisation a été sollicitée.

Ces chiffres montrent une répartition du contentieux tournée pour l'essentiel vers la réparation des préjudices individuels, tandis que l'action pénale et la voie administrative n'apparaissent que résiduelles. Ces éléments de nature quantitative relatifs aux litiges sont corroborés par l'étude des décisions concernées : la logique individuelle des litiges apparaît alors nettement, que ce soit dans les litiges portés devant les juridictions civiles, répressives ou administratives.

8. $2^{\mathrm{e}}$ chambre civile (Civ. $2^{\mathrm{e}}$ ), 19 septembre $2013, \mathrm{n}^{\mathrm{o}} 12-21.350 ;$ Civ. $^{\mathrm{e}}, 3$ avril 2014, $\mathrm{n}^{\mathrm{o}}$ 13-14.863. 
Entre égalité de traitement et harcèlement, quel fondement juridique de la discrimination syndicale?

TABLEAU 1 - État du contentieux devant la Cour de cassation et les cours d'appel, le Conseil d'État et les juridictions administratives, 2012-2014

\begin{tabular}{lc}
\hline & $\begin{array}{c}\text { Nombre de décisions relatives } \\
\text { à la discrimination syndicale }\end{array}$ \\
\hline $\begin{array}{l}\text { Cour de cassation, chambres civiles : } \\
\text { chambre sociale } \\
\text { chambre criminelle (litiges répressifs) }\end{array}$ & 253 \\
$2^{\mathrm{e}}$ chambre civile & 236 \\
\hline $\begin{array}{l}\text { Cours d'appel } \\
\quad \text { dont conseil de prud'hommes (litiges individuels du travail) }\end{array}$ & 2 \\
\hline Conseil d'État & 1243 \\
Cours administratives d'appel & 1198 \\
\hline
\end{tabular}

Champ : décisions comportant en plein texte l'expression « discrimination syndicale » entre le $1^{\text {er }}$ janvier 2012 et le 31 décembre 2014. Source : Lamyline (www.lamyline.fr).

Cette répartition quantitative montre que les contentieux sont, dans leur vaste majorité, engagés devant les juridictions civiles. Comme nous le verrons ensuite, les contentieux administratifs et répressifs jouent néanmoins un rôle complémentaire important dans la garantie des droits syndicaux. Il convient enfin d'être attentif à l'importance des stratégies collectives dans une partie de ces contentieux, à travers l'étude des décisions sérielles (rendues en série contre un même employeur) que nous analyserons en dernier.

\section{La quasi-totalité des affaires est constituée par un contentieux civil de la réparation des conséquences de la discrimination}

L'essentiel des contentieux impliquant les discriminations syndicales est constitué d'affaires prud' homales visant à obtenir réparation d'actes ou de pratiques jugés discriminatoires. Ces contentieux paraissent relativement abondants, au moins par rapport aux autres motifs de discrimination (sexe, origine, âge, etc.). Le volume contentieux paraît néanmoins modeste au regard de l'ensemble des questions posées par les désavantages subis par les représentants du personnel et les militants syndicaux. Le nombre d'affaires en appel impliquant des discriminations syndicales paraît se situer à un niveau maximal de 1198 entre 2012 et 2014, soit environ 400 affaires par an, ce qui, en tenant compte d'un taux d'appel d'environ $65 \%$ en moyenne pour les décisions prud'homales (GUILLONEAU, SERVERIN, 2015), indiquerait un volume contentieux au niveau des prud'hommes d'environ 660 décisions par an. Encore ce nombre se situe-t-il dans une fourchette haute, car les discriminations syndicales dans ces litiges ont, comme nous le verrons, une place très variable. Ce volume contentieux doit être rapporté à une population estimée à 767000 représentants élus ou désignés (PAK, PignONI, 2014) et à 1,5 million de militants dans le secteur privé (PIGNONI, 2016), ainsi qu'à la persistance de pratiques discriminatoires très répandues envers les représentants du personnel (OBSERVATOIRE DE LA DISCRIMINATION ET DE LA RÉPRESSION SYNDICALES, 2014). Les désavantages subis par les représentants du personnel ou militants semblent ainsi n'être que rarement soumis aux juridictions, et donner lieu en majorité à d'autres 
types de résolution des différends, notamment le recours à l'intervention de l'inspection du travail ou au défenseur des droits, ou ne faire l'objet d'aucune contestation.

Ces données générales résultent de la seule interrogation de la base de données des arrêts des cours d'appel ou de la Cour de cassation. Les autres caractéristiques, plus qualitatives, exigent un travail plus systématique de lecture et de codage des arrêts, que nous avons effectué sur la base d'un échantillon plus réduit des 124 arrêts rendus par les cours d'appel sur l'ensemble du territoire, au cours du dernier trimestre 2014.

De façon générale, les affaires montrent des caractéristiques communes, qui reflètent l'état général du contentieux social (MunOz PEREZ, SERVERIN, 2005). Elles proviennent dans leur totalité de demandes de salariés. Les litiges ont tous été tranchés en première instance par un conseil de prud'hommes, à l'exception d'une seule affaire tranchée par un tribunal de grande instance (TGI) ${ }^{9}$. L'analyse des décisions est intéressante pour repérer la place des affaires dites « sérielles » dans ces contentieux. Celles-ci opposent, à travers des litiges individuels, différents salariés confrontés à une même difficulté face à une entreprise (par exemple, un retard de carrière subi par plusieurs de ses représentants du personnel). De telles affaires n'occupent qu'une place limitée dans l'échantillon considéré : seules quatre séries peuvent être repérées.

Les syndicats sont très peu présents dans ces litiges, malgré l'importance des enjeux, les pratiques discriminatoires ayant des répercussions probables sur les adhésions des salariés et leur implication dans les institutions représentatives du personnel. Sur les 124 décisions étudiées, les syndicats sont partie principale dans une seule décision ${ }^{10}$. Ils ne sont intervenus dans une action engagée par un salarié que dans 11 affaires. Les syndicats de la Confédération générale du travail (CGT) semblent les plus prompts à utiliser cette voie d'action, étant présents dans sept affaires, tandis que la Confédération française démocratique du travail (CFDT) s'est jointe à deux actions, SUD à une action, de même que le syndicat du livre et un « Syndicat des employés mécontents ${ }^{11}$ ».

À l'instar du reste du contentieux, l'action de substitution, par laquelle les syndicats peuvent introduire des litiges au nom et pour le compte des salariés, n'est pratiquement pas mobilisée par les syndicats à l'exception d'une seule décision ${ }^{12}$.

Enfin, l'objet des demandes est très majoritairement la réparation pécuniaire des conséquences des actes discriminatoires. Seules deux décisions sur l'échantillon du dernier trimestre 2014 demandent une réintégration pour discrimination syndicale ${ }^{13}$, et cinq invoquent la nullité du licenciement sans demander la réintégration (voir tableau 2).

9. SUD contre Air France, Paris, 27 octobre 2014, n ${ }^{\circ}$ 14/07354.

10. Ibid.

11. Paris, 31 janvier 2013, $\mathrm{n}^{\circ} 11 / 01244$.

12. Grenoble, 25 novembre 2014, n ${ }^{\circ}$ 13/03004, Caterpillar c. Syndicat Symetal 38.

13. Paris, 27 novembre 2014, $\mathrm{n}^{\circ}$ 13/00150 et Rennes, 29 octobre 2014, $\mathrm{n}^{\circ}$ 12/03613. 
TABLEAU 2 - Principales caractéristiques des litiges civils devant les cours d'appe ${ }^{14}$

\begin{tabular}{|c|c|}
\hline Litiges portant exclusivement sur les discriminations & 39 \\
\hline $\begin{array}{l}\text { Objets des demandes : } \\
\text { inégalités de carrière } \\
\text { inégalités salariales } \\
\text { préjudice causé par un acte discriminatoire } \\
\text { communication de pièces }\end{array}$ & $\begin{array}{r}18 \\
10 \\
8 \\
1\end{array}$ \\
\hline $\begin{array}{l}\text { Résultats des actions : } \\
\text { satisfaction (totale ou partielle) } \\
\text { rejet de la demande } \\
\text { non statué sur le fond }\end{array}$ & $\begin{array}{r}26 \\
10 \\
3\end{array}$ \\
\hline $\begin{array}{l}\text { Intervention des syndicats dans les litiges: } \\
\text { CGT } \\
\text { SUD }\end{array}$ & $\begin{array}{l}5 \\
2 \\
\end{array}$ \\
\hline Litiges portant sur des discriminations et d'autres objets & 13 \\
\hline $\begin{array}{l}\text { Objets des autres demandes: } \\
\text { harcèlement } \\
\text { contestation du licenciement } \\
\text { contestation d'une sanction disciplinaire } \\
\text { réparation des conséquences d'une faute inexcusable } \\
\text { rappel de salaire }\end{array}$ & $\begin{array}{l}6 \\
3 \\
2 \\
1 \\
1\end{array}$ \\
\hline $\begin{array}{l}\text { Résultat des actions portant sur la discrimination : } \\
\text { satisfaction (totale ou partielle) } \\
\text { rejet de la demande }\end{array}$ & $\begin{array}{l}5 \\
8\end{array}$ \\
\hline $\begin{array}{l}\text { Intervention des syndicats dans les litiges : } \\
\text { CFDT } \\
\text { CGT }\end{array}$ & $\begin{array}{l}1 \\
1 \\
\end{array}$ \\
\hline Litiges dans lesquels la place de la discrimination est plus incertaine & 76 \\
\hline $\begin{array}{l}\text { Objet principal du litige : } \\
\text { licenciement } \\
\text { rappel de salaire ou de primes } \\
\text { objets de demandes multiples } \\
\text { prise d'acte de la rupture } \\
\text { sanction disciplinaire } \\
\quad \text { dont litige contre la Caisse des écoles de Nouméa } \\
\text { harcèlement } \\
\text { résiliation judiciaire du contrat de travail } \\
\text { faute inexcusable }\end{array}$ & $\begin{array}{r}15 \\
13 \\
9 \\
6 \\
24 \\
20 \\
5 \\
3 \\
1\end{array}$ \\
\hline $\begin{array}{l}\text { Résultat de ces actions en matière de discrimination : } \\
\text { satisfaction (totale ou partielle) } \\
\text { rejet de la demande } \\
\text { non statué sur le fond } \\
\text { absence de demande spécifique } \\
\text { hors champ des discriminations syndicales }\end{array}$ & $\begin{array}{r}11 \\
30 \\
1 \\
22 \\
12\end{array}$ \\
\hline $\begin{array}{l}\text { Intervention des syndicats dans les litiges : } \\
\text { CGT } \\
\text { CFDT }\end{array}$ & $\begin{array}{l}2 \\
1 \\
\end{array}$ \\
\hline Total & 124 \\
\hline
\end{tabular}

Champ : décisions rendues par les cours d'appel, du $1^{\text {er }}$ octobre 2014 au 31 décembre 2014.

Source : Lamyline (www.lamyline.fr).

14. Litiges portés devant les juridictions civiles en appel, tranchés en première instance soit par les conseils de prud'hommes (litiges individuels) soit par les tribunaux d'instance (litiges collectifs). 
L'analyse de ces décisions montre qu'elles procèdent de trois formes d'action différentes, selon que l'objet de la demande porte sur la seule discrimination syndicale, sur une pluralité d'objets dont la discrimination syndicale, ou s'ils portent sur des objets moins bien identifiés au regard de la problématique des discriminations syndicales (tableau 2).

Certains litiges ont pour objet exclusif les discriminations. Ils concernent un peu moins d'un tiers de l'échantillon étudié (39 sur les 124 affaires analysées en détail). Ils portent sur la discrimination subie dans la carrière (18 décisions) ou les inégalités salariales (10 décisions). Ils nécessitent alors des comparaisons réalisées à partir de panels et se réduisent souvent à cette seule question, les salariés ne cherchant pas à montrer le lien entre la différence pratiquée et le motif discriminatoire poursuivi par l'employeur.

À côté de ces litiges concernant des questions d'égalité dans les carrières et les rémunérations, on trouve huit affaires concernant des réparations de faits de discrimination, plus clairement orientées vers la sanction des discriminations syndicales, que celles-ci visent l'exercice des mandats représentatifs ou le fait d'être militant syndical.

Quoi qu'il en soit, ces différents litiges ont un objet clairement centré sur la question des discriminations syndicales. Cette caractéristique influe sans doute sur l'implication des syndicats dans ces dossiers, qui, dans sept des 39 affaires concernées (soit $18 \%$ du sous-échantillon) sont intervenus à l'instance au nom de la défense de l'intérêt collectif de la profession et en demandant parfois des dommages et intérêts à ce titre.

Pour ce qui est des résultats, ces affaires conduisent dans une large majorité à faire droit à la demande des salariés, puisque 26 des 36 affaires (soit $72 \%$ du sous-échantillon) ayant statué sur le fond leur octroient une satisfaction totale ou partielle, ce qui est comparable au taux de succès des demandes devant les conseils de prud'hommes (Munoz Perez, Serverin, 2005). L'analyse montre que ces actions s'appuient sur un dossier ayant de sérieuses chances d'aboutir grâce à une maitrise des instruments juridiques d'action en justice par les justiciables concernés, qui savent prendre appui sur des réseaux d'expertise (avocats spécialisés, syndicats). Le taux de réussite en appel est cependant à relativiser, car il ne permet pas d'observer les abandons de procédure en cours de litige, très nombreux dans les affaires prud'homales.

D'autres litiges (13), clairement rapportés à des actions fondées sur les discriminations syndicales, ont pour particularité de cumuler les objets. Ils permettent de comprendre que les actes discriminatoires reçoivent rarement l'unique qualification de «discrimination» : sont en cause des sanctions disciplinaires, licenciements ou harcèlements, dont le salarié conteste la légitimité en prétendant notamment qu'ils sont motivés par un mobile discriminatoire. En proportion, ce sont ici les pratiques de harcèlement qui semblent en majorité contestées (six affaires, sur les treize du sous-échantillon), devant les licenciements (trois affaires). Viennent ensuite de façon plus anecdotique la contestation de sanctions disciplinaires, des rappels de salaire ou la recherche d'indemnisation des conséquences d'une faute inexcusable. L'invocation 
de la discrimination dans un tel contexte peut paraître plus diluée, ce qui explique des taux de satisfaction de ces demandes très inférieurs aux moyennes habituellement observées dans les litiges du travail. Huit demandes sur treize sont ici rejetées, soit un peu plus de $60 \%$, encore que le nombre d'affaires soit trop peu significatif pour en tirer des enseignements généraux. Cette proportion pourrait s'expliquer par la place occupée par le harcèlement dans l'échantillon considéré. Objet de litige assez difficile à cerner juridiquement, il ne parait pas souvent aboutir favorablement devant les juridictions civiles.

Enfin, une majorité d'affaires ( 76 sur 124, soit $61 \%$ de l'échantillon des décisions du dernier trimestre 2014) se réfère aux discriminations syndicales, mais de façon beaucoup plus diffuse, de sorte que la discrimination ne constitue qu'un objet lointain. Les configurations de ces affaires sont assez diverses. Une partie d'entre elles s'avère sans lien avec la discrimination syndicale, soit qu'elle ait fait l'objet d'un litige antérieur simplement rappelé, soit qu'elle ait simplement été évoquée dans les moyens des parties sans faire l'objet ni de demande ni d'une véritable argumentation. Dans l'échantillon observé, 12 affaires n'ont aucun rapport avec les discriminations syndicales, et 22 mobilisent la discrimination comme moyen au soutien d'une demande principale, sans faire l'objet d'aucune demande propre.

Dans ce sous-échantillon, les demandes principales ont porté sur le licenciement (15 affaires sur 76) ou sur des rappels de salaires (13 affaires). Les salariés font valoir ici que le licenciement est injustifié, notamment en ce qu'il se fonde sur des mobiles discriminatoires, mais sans que cela conduise à la formulation de demandes spécifiques portant sur des discriminations. De même, les salariés, à l'appui d'un litige salarial, se contentent parfois de mentionner que l'absence de versement d'une rémunération due provient de ce mobile. Les litiges peuvent aussi porter sur une demande d'annulation de sanction disciplinaire, dont le mobile serait discriminatoire (25 décisions). Leur présence quantitativement importante dans le sous-échantillon provient d'une série de 20 décisions de la cour d'appel de Nouméa ${ }^{15}$, auprès de laquelle était contestée la mise à pied de salariés au cours d'un mouvement de grève estimé illicite par l'employeur. Enfin, il faut signaler la place importante des litiges portant sur la rupture du contrat de travail en dehors du licenciement : six litiges sont fondés sur la prise d'acte de la rupture, et trois sur des demandes de résiliation judiciaire des contrats de travail. Les salariés font valoir dans ces litiges que ces actes discriminatoires peuvent justifier la rupture aux torts de l'employeur.

Dans ces différentes affaires, la discrimination syndicale demeure un objet de litige, mais elle apparait secondaire au regard d'autres objets (parfois assez nombreux), ou n'apparaît qu'au titre d'un simple argument. En dehors des 12 affaires repérées comme extérieures au champ des discriminations, les autres affaires peuvent néanmoins être identifiées comme des formes particulières de litiges portant sur les discriminations. Elles permettent de comprendre que ceux-ci ne se présentent pas 
de façon majoritaire sous la forme spécifique que leur confèrent les articles L. 1132-1 et suivants du Code du travail. Les discriminations se manifestent le plus souvent à l'occasion de la contestation de décisions de l'employeur: sanction disciplinaire, rupture du contrat de travail, changement de conditions de travail ou modification contractuelle, pratiques salariales inégalitaires, etc. Il est dès lors compréhensible et inévitable que les catégories juridiques soient invoquées simultanément et de façon plus ou moins mêlée. Les faits et les préjudices causés étant souvent les mêmes, on assiste à une forme de fongibilité des actions qui laisse une grande liberté aux parties dans la construction de l'édifice litigieux (GuIOMARD, 2013).

Cette dilution des contentieux relatifs aux discriminations syndicales dans d'autres objets de litige rend difficile l'évaluation de l'état des contentieux. Certaines actions, menées sur le terrain de l'égalité de traitement ou de la contestation de la cause de licenciement ou des sanctions disciplinaires, peuvent permettre de résoudre des différends trouvant leur origine dans une discrimination syndicale : ce n'est que lorsque le salarié souhaite obtenir la nullité du licenciement et la réintégration dans l'entreprise que les discriminations sont explicitement invoquées. Bien que ce type de demande apparaisse peu dans les contentieux, des différends portant sur les discriminations peuvent ainsi ne pas comporter de demande spécifique portant sur la réparation des conséquences d'une discrimination et mentionner celle-ci à titre d'argument au soutien d'un moyen principal, voire dans certains litiges, n'y porter aucune référence.

\section{Les contentieux répressifs et administratifs, garants du respect des droits syndicaux}

Si le contentieux prud'homal est quantitativement le plus important, il n'épuise pas le spectre des actions en justice possibles. Des actions peuvent également être menées devant les autres ordres de juridiction. Elles paraissent plus difficiles à mener, tant par la délimitation étroite des objets de litiges que par l'absence d'aménagement adéquat des règles de preuve.

L'étude des décisions rendues dans la période récente révèle l'utilité de ces contentieux pour préserver le respect des prérogatives confiées aux représentants du personnel, qui est l'une des dimensions des discriminations syndicales. Ces décisions permettent à la fois de garantir le respect du statut protecteur des représentants du personnel dans l'ordre administratif, et d'en assurer une ferme sanction dans l'ordre répressif.

Le contentieux administratif des discriminations se révèle assez peu abondant : seules 210 décisions de cours administratives d'appel, rendues de 2012 à 2014 , se réfèrent à la « discrimination syndicale ${ }^{16}$ » ou au « caractère discriminatoire » du licenciement des salariés protégés.

16. Interrogation des bases de données par l'expression « discrimination syndicale ». 
La jurisprudence du Conseil d'État montre assez clairement la place occupée par ces discriminations dans le contrôle des autorisations de licenciement. La haute juridiction indique que l'autorité administrative doit contrôler que le licenciement n'est pas en « rapport avec les fonctions représentatives normalement exercées ou l'appartenance syndicale de l'intéressé », et qu'elle doit vérifier « si les éléments à l'appui de la demande justifient [le licenciement] compte tenu de la nature des fonctions exercées par le salarié, de l'ensemble des règles applicables à son contrat de travail et des exigences propres à l'exécution du mandat dont il est investi ${ }^{17} \gg$. Le contrôle administratif des licenciements a donc pour finalité principale de vérifier que le licenciement n'a pas de rapport avec l'appartenance syndicale ou l'exercice du mandat : il permet « d'éviter que ces salariés ne fassent l'objet de mesures discriminatoires dans le cadre d'une procédure de licenciement ou de transfert partiel d'entreprise ${ }^{18} »$.

Le «statut protecteur » est donc orienté vers la prévention des discriminations syndicales. Toutefois, le contrôle juridictionnel semble le plus souvent se limiter à vérifier que l'employeur dispose de raisons objectives de licencier le salarié, le régime probatoire appliqué ne lui imposant pas de justifier de l'absence de discrimination. Les juridictions n'appliquent pas le raisonnement en deux temps en vigueur devant les juridictions civiles : celui-ci impose dans un premier temps que le demandeur établisse des éléments de fait laissant supposer l'existence d'une discrimination, qu'il revient dans un second temps au défendeur de contester en prouvant que sa décision est justifiée par des éléments objectifs étrangers à toute discrimination (art. L. 1134-1, Code du travail). Aussi l'absence de discrimination est-elle le plus souvent déduite du constat de l'existence d'une raison objective pour licencier le salarié. Le Conseil d'État se contente en effet, en présence d'un tel motif, de rejeter les demandes invoquant une discrimination syndicale par une formule laconique : « la discrimination invoquée par le requérant n'est pas établie par le dossier ${ }^{19}{ }$. Ce même raisonnement est appliqué pour les différents motifs de licenciement ${ }^{20}$ et pour les actes autres que le licenciement (à l'instar des transferts d'entreprise ${ }^{21}$ ).

Ce contrôle, assez peu approfondi, de l'absence de discrimination syndicale se reflète dans les décisions rendues par les cours administratives d'appel, qui pourtant contrôlent et le motif invoqué et l'absence de lien avec le mandat. Mais, tout comme le Conseil d'État, elles ne paraissent pas pousser très loin les investigations à ce sujet, se limitant à indiquer qu'il «ne ressort pas des pièces » que l'employeur ait tenté d'entraver l'exercice du mandat ${ }^{22}$. Certaines décisions témoignent d'un niveau d'exi-

17. Conseil d'État (CE), $1^{\text {er }}$ avril $1992, n^{\circ} 118580$, Société Ladbroke Hotels France, publié au recueil Lebon.

18. CE, 28 mars $2013, n^{\circ} 350436$, mentionné dans les tables du recueil Lebon.

19. CE, Société Ladbroke Hotels France, doc. cité.

20. Par exemple pour le contrôle des autorisations de licenciement en cas de licenciement pour motif économique :

CE, 8 avril 2013, $\mathrm{n}^{\circ} 348559$, publiée au recueil Lebon.

21. CE, 10 mars 1997, $n^{\circ} 169830$, publiée au recueil Lebon et CE, 28 mars 2013, n 350436, mentionné dans les tables du recueil Lebon.

22. Cour administrative d'appel (CAA), Nancy, 2 juin 2014, $n^{\circ} 13$ NC00850. Cette répartition de la preuve semble conforme aux indications du Conseil d'État qui indique qu'il revient au juge d'appel « de se forger sa conviction au vu 
gence accru. Une affaire montre ainsi la volonté de contrôler les raisons de la demande d'autorisation au-delà de la faute du représentant (absences injustifiées et propos tenus dans un tract) pour vérifier l'absence de lien de cette faute avec le mandat ${ }^{23}$. Le contrôle de l'absence de discrimination fait donc l'objet d'un deuxième temps du raisonnement, après le contrôle de l'existence d'une faute par le représentant. Sur le terrain de la preuve, deux arrêts dénotent une certaine audace, en se référant aux dispositions du Code du travail sur la preuve des discriminations, qui exigent de l'employeur de prouver que sa décision est justifiée par des éléments objectifs étrangers à toute discrimination lorsque le salarié présente des éléments de fait laissant supposer l'existence d'une discrimination. Les juges vérifient alors dans le dossier de demande d'autorisation que l'employeur a bien justifié l'absence de discrimination ${ }^{24}$.

Le statut protecteur permet ainsi un contrôle de l'absence de discrimination en cas de licenciement d'un salarié protégé mais les règles de preuve le rendent assez fragile.

Précisons par ailleurs que le contentieux administratif des salariés protégés ne peut pas être conçu de façon isolée. Les juridictions administratives ont pour seul pouvoir d'autoriser ou de refuser le licenciement et ne peuvent se prononcer sur d'autres aspects du contrôle des licenciements ${ }^{25}$ (critères d'ordre du licenciement ${ }^{26}$, légèreté blâmable en cas de cessation d'activité ${ }^{27}$, éléments postérieurs à l'autorisation de licenciement ${ }^{28}$ ) ou encore de sanctionner la méconnaissance par l'employeur des refus d'autorisation. Le contentieux civil sur les discriminations syndicales fournit en ce sens quelques exemples d'actions en réintégration de salariés protégés, licenciés sans autorisation ou au mépris d'un refus de licenciement par l'inspection du travail, ou d'une autorisation de licenciement annulée par le juge administratif ${ }^{29}$. Ces décisions permettent ainsi de sanctionner fermement la méconnaissance du statut protecteur sans que la question de la discrimination ait véritablement à être débattue.

Pour ce qui est enfin du contentieux répressif, celui-ci s'avère difficile à évaluer, car les décisions des cours d'appel en matière répressive ne sont pas versées sur la base de données Jurica, pour des raisons relatives à la protection de la vie privée. Il n'est donc possible d'avoir une idée de ces contentieux répressifs qu'à travers les décisions de la chambre criminelle de la Cour de cassation. Seules 15 affaires concernant les discriminations syndicales ont été rendues dans la période allant de 2012 à 2014 (à comparer avec les 253 affaires repérées en matière prud'homale).

des échanges contradictoires des parties sans faire reposer la charge de la preuve sur le seul salarié » (CE, 10 décembre 2014, n ${ }^{\circ} 359179$, inédit).

23. CAA, Versailles, 20 mai 2014, $\mathrm{n}^{\circ} 13$ VE01484.

24. CAA, Nantes, 27 septembre 2012, $\mathrm{n}^{\circ} 11$ NT02686 ; CAA, Douai, 14 juin $2012 \mathrm{n}^{\circ}$ 11DA00092. Les deux affaires portent sur l'application des critères d'ordre des licenciements pour motif économique.

25. CE, 8 avril 2013, doc. cité.

26. Chambre sociale (Soc.), 27 octobre $2004, \mathrm{n}^{\circ} 02-46.935$.

27. CE, 8 avril 2013, doc. cité.

28. Soc., 10 février $1999, \mathrm{n}^{\circ} 93-43.561$.

29. Voir Nancy, 17 octobre 2014, $\mathrm{n}^{\circ}$ 12/01373 (licenciement suite à un refus d'autorisation) ; Versailles, 14 mai 2014, $\mathrm{n}^{\circ}$ 12/05167 (licenciement sans demande d'autorisation) ; Rennes, 29 octobre 2014, $\mathrm{n}^{\circ}$ 12/03613 et Paris, 27 mai 2014, $\mathrm{n}^{\mathrm{o}}$ 09/06739 (réintégration suite à l'annulation d'une autorisation). 
L'étude de ces décisions montre néanmoins l'utilité de ces contentieux répressifs, essentiellement comme mode de garantie de l'exercice des prérogatives d'action reconnues aux représentants du personnel. Aucune décision n'a été rendue sur le fondement de l'article 225-1 du Code pénal (prohibition des actions fondées sur les activités syndicales), et toutes celles qui statuent sur la discrimination syndicale prennent appui sur les articles L. 2141-5 et/ou L. 2146-2 du Code du travail, ce qui suggère que le texte est davantage utilisé pour sanctionner les atteintes au fonctionnement des institutions syndicales que comme un moyen de réparer des préjudices subis personnellement par les militants et les représentants du personnel.

Différents types d'affaires semblent se distinguer. Dans les cas les plus nombreux, le salarié saisit le tribunal, soit par voie de plainte avec constitution de partie civile, soit par la voie de la citation directe, pour discrimination syndicale et entrave à l'exercice du droit syndical simultanément. La discrimination semble utilisée ici à fin de sanction à l'encontre des comportements les plus hostiles aux syndicats. Les arrêts en fournissent des exemples : favoritisme marqué à l'égard d'une obédience syndicale donnée ${ }^{30}$, refus de réintégrer un salarié protégé licencié sans autorisation ${ }^{31}$, exercice de pressions et brimades diverses ${ }^{32}$. Ces différentes affaires montrent clairement à la fois l'intérêt de la sanction pénale des discriminations syndicales et le lien étroit qui l'unit au délit d'entrave aux fonctions des représentants du personnel.

La jurisprudence de la Cour de cassation met aussi en évidence les limites de ce droit en matière de preuve. La haute juridiction casse par exemple une décision par laquelle les juges du fond avaient condamné l'employeur pour discrimination syndicale d'un salarié qui avait, sans explication possible, subi une série de désavantages (privation d'accès au réseau informatique et aux informations internes à l'entreprise, changement de fonctions). Pour les juges du fond, le lien probable avec les fonctions syndicales suffisait à la constitution du délit. La Cour estime qu'ils ont renversé la charge de la preuve : il leur appartenait de « rechercher l'existence d'une relation de causalité entre les mesures jugées discriminatoires et l'appartenance ou l'activité syndicale de la partie poursuivante ${ }^{33} »$.

Cette rigueur dans la preuve des discriminations, inhérente au droit pénal, est parfois contrebalancée par des décisions plus audacieuses. Un arrêt très intéressant du 14 janvier 2014 semble ainsi admettre, pour la première fois en matière pénale, que l'administration de la preuve sur la base de panels de comparaison peut être suffisante pour permettre une condamnation du chef d'entreprise pour discrimination syndicale. L'inspecteur du travail avait ici procédé à une analyse comparative des données sur l'évolution des carrières des représentants du personnel, méthode qui, pour les juges du fond, permettait de « démontrer de manière rigoureuse et fiable l'existence d'une discrimination ». La Cour de cassation rejette le pourvoi contre cette décision, estimant

30. Chambre criminelle (Crim.), 19 novembre 2013, $\mathrm{n}^{\circ} 12-82.163$, inédit.

31. Crim., 28 mai 2013, $\mathrm{n}^{\circ} 12-81.468$, Bulletin criminel, $\mathrm{n}^{\circ} 118$.

32. Crim., 11 décembre 2012, n 11-88.114, inédit.

33. Crim., 19 février 2013, $\mathrm{n}^{\circ}$ 12-81.925, inédit. 
que les juges du fond avaient pu souverainement apprécier, à partir des éléments de preuve présentés, que les dirigeants de la société avaient mis en œuvre des mesures discriminatoires ${ }^{34}$.

L'utilité des procédures pénales est également attestée par leur complémentarité avec les actions révélées par l'étude des décisions des juridictions civiles. Certaines affaires (au nombre de 36 de 2012 à 2014) font référence à des poursuites pénales engagées par le salarié. Elles peuvent porter sur les mêmes faits que ceux qui font l'objet de l'action prud'homale, le caractère intentionnel du délit en matière pénale n'excluant pas l'action devant les conseils de prud' hommes ${ }^{35}$, ou sur des faits distincts tels que la condamnation passée de l'employeur pour harcèlement moral ou pour entrave, présentée comme élément de preuve d'une discrimination syndicale ${ }^{36}$. Par ailleurs, quelques affaires montrent la pugnacité des représentants du personnel face à des comportements discriminatoires répétés de certaines entreprises. La multiplicité des voies d'action est alors sans doute le gage de la préservation des droits des salariés ${ }^{37}$.

L'analyse de ces différentes décisions montre au total un contentieux assez varié de la discrimination syndicale, dont la finalité apparaît de façon prépondérante comme individuelle : elles permettent au salarié discriminé d'obtenir une réparation à titre personnel. Cette perspective ne serait pas complète si elle ignorait la dimension collective propre à certaines affaires qui, certes moins nombreuses, revêtent une importance toute particulière pour la collectivité des salariés.

\section{La présence de séries de décisions, révélatrice de stratégies d'action collectives}

La problématique des discriminations ne saurait être purement individuelle. Les salariés discriminés dans leur carrière ou subissant des désavantages destinés à limiter leur action représentative le sont rarement de façon isolée, les pratiques d'entreprises visant souvent l'ensemble des représentants du personnel ou au moins une partie d'entre eux.

Les outils procéduraux à la disposition des syndicats ne paraissent pas garantir une sanction efficace de ces discriminations, entendues en un sens collectif. La jurisprudence de la Cour de cassation se montre ouverte à la prise en compte de l'intérêt collectif de la profession (art. L. 2132-3 du Code du travail) pour fonder des actions collectives devant les juridictions ${ }^{38}$. Toutefois, ces actions ne peuvent permettre de

\footnotetext{
34. Crim., 14 janvier 2014, n 12-85.091, inédit.

35. Besançon, 3 février 2012, $\mathrm{n}^{\circ} 11 / 00470$.

36. Montpellier, 4 décembre 2013, $\mathrm{n}^{\circ}$ 12/03545 ; Versailles, 6 juin 2013, n 10/03230.

37. Pau, 16 mai 2013, n 11/02306 : une salariée, licenciée de son emploi, est embauchée par le comité d'entreprise. L'employeur saisit le TGI pour obtenir l'annulation de cette embauche. La salariée se prévaut ensuite de cette action et de la condamnation pour diffamation de son employeur dans une affaire correctionnelle la concernant à l'appui de sa demande de reconnaissance d'une discrimination syndicale.

38. Soc., 9 février 2000, $\mathrm{n}^{\circ} 97-40724$, Bulletin civil $V, \mathrm{n}^{\circ} 58$ pour l'action devant les juridictions civiles. Pour une constitution de partie civile pour agir devant les juridictions répressives, cf. Crim., 29 octobre 1996, ${ }^{\circ}$ 94-85.028, Bulletin criminel, $\mathrm{n}^{\circ} 379$ : «Le fait de licencier un salarié en raison de son appartenance à un syndicat ou de l'exercice
} 
reconnaitre directement des droits au profit des salariés victimes sans que ceux-ci engagent à leur tour un litige ou soient partie personnellement à l'action. Les actions de substitution, qui permettraient de créer de tels droits au profit des salariés, ne sont pas perçues par les syndicalistes, de par leurs modalités, comme un mode d'action satisfaisant (COHEN, 1990, PetiT, 2004) ${ }^{39}$.

Les syndicats semblent dès lors envisager l'action collective par une utilisation, avec le concours des militants, de l'action individuelle en justice. Ils participent à la constitution des preuves, à la détermination des argumentaires et à la construction des dossiers avec le concours d'avocats spécialisés, laissant aux salariés individuellement le soin de réclamer devant les juridictions la réparation des discriminations. Les syndicats se joignent souvent à ces actions et interviennent dans ces litiges au nom de la défense de l'intérêt collectif de la profession, en réclamant des dommages et intérêts afin d'obtenir réparation du préjudice que leur cause la discrimination. Mais ils ne le font pas systématiquement, préférant parfois laisser agir seuls leurs adhérents. Derrière ces actions, qui apparaissent dans le contentieux sous forme de décisions individuelles, on trouve donc une forme particulière de l'action syndicale, qui peut avoir des finalités variées au-delà de la réparation des discriminations individuelles : stratégie de négociation d'accords sur l'exercice du droit syndical, modification du rapport de force avec un chef d'entreprise, etc.

Ces affaires sont assez difficiles à repérer dans le contentieux car les syndicats ne sont pas explicitement présents. Il est toutefois assez aisé d'observer une partie d'entre elles à partir des séries de décisions. Celles-ci sont le produit de saisines individuelles multiples, formulant des demandes similaires sur la base d'un argumentaire commun construit par les syndicats.

Pour identifier la part prise par ces actions sérielles, nous avons procédé à une recherche des arrêts de cours d'appel portant sur la question de la discrimination syndicale et avons systématiquement relevé le nom des entreprises défenderesses, pour la période 2012-2014. Nous avons considéré qu'une série commençait à partir de trois affaires.

Il apparaît alors que ces actions collectives sont relativement peu nombreuses : 170 affaires sur les trois années concernaient le même défendeur. Rapporté à un nombre de 1243 affaires, le nombre de séries représenterait ainsi $14 \%$ des litiges.

Les stratégies à l'origine de ces affaires et les résultats auxquels elles aboutissent sont variables. Une grande partie d'entre elles concerne des discriminations dans la carrière ou la rémunération. Il s'agit des litiges numériquement les plus importants, opposant des représentants du personnel ou anciens représentants du personnel aux caisses de sécurité sociale (38 affaires repérées). Ils sont introduits par des militants

d'une activité syndicale est, en lui-même, générateur d'un préjudice subi par la profession à laquelle appartient l'intéressé et dont les syndicats qui représentent cette profession peuvent demander réparation. »

39. En effet, ces actions permettent aux employeurs d'identifier les salariés et, s'ils le souhaitent, de mettre individuellement fin aux instances engagées par les syndicats, ce qui peut se traduire par des pressions sur ces salariés et des syndicats désavoués dans leur action. 
de la CGT, disposant d'une ancienneté importante (souvent plus de trente ans) et, pour nombre d'entre eux, partis à la retraite. Se fondant explicitement sur la constitution de «panels » de comparaison établis avec des salariés embauchés à la même date, ils invoquent des discriminations dans le déroulement de la carrière. Les approches variées suivies par les différentes cours d'appel montrent bien les tensions qui traversent le droit des discriminations. Certaines juridictions, comme la cour d'appel de Versailles, font largement droit aux demandes, avec des réparations importantes allant de 19000 à 250000 euros. Elle estime que les échantillons produits sont dotés d'une « valeur représentative » fiable et laissent présumer une discrimination sans que l'employeur justifie d'un motif objectif de différence ${ }^{40}$.

Mais d'autres juridictions ne se basent pas sur la seule comparaison des rémunérations et scrutent de façon plus détaillée les évolutions de carrière. La cour d'appel de Douai, par exemple, infirme les décisions des premiers juges qui avaient fait droit aux mêmes demandes, estimant que les salariés avaient bénéficié à la fois d'un égal accès à la formation et d'accords successifs sur la carrière des représentants du personnel ayant déjà permis un rattrapage salarial ${ }^{41}$. D'autres se fient à un contre-panel produit par l'entreprise et jugé plus fiable ${ }^{42}$.

Ces séries montrent ainsi, aux côtés des actions à finalité individuelle, largement majoritaires, une part minoritaire de litiges ayant une perspective plus collective engagée avec le soutien des syndicats.

La variété des litiges engagés sur le fondement de la discrimination syndicale étant ainsi caractérisée, il convient d'en souligner la fréquente ambivalence.

\section{L'ambiguiité des actions fondées sur la discrimination syndicale}

Les règles du Code du travail semblent construire la discrimination syndicale sur un modèle unique et simple par lequel le salarié combattrait un acte motivé par un but discriminatoire ou ayant un tel effet. Pourtant, la structure même du droit des discriminations syndicales, orienté à la fois vers une protection de l'exercice des fonctions syndicales et vers la protection des droits des personnes, n'est pas sans conférer une certaine ambivalence aux contentieux dans lesquels ces règles sont invoquées et appliquées. Elle se retrouve dans les raisonnements déployés, qui orientent les parties et les juges sur le seul terrain de la comparaison, engageant les litiges sur le terrain de l'égalité de traitement davantage que sur celui de la discrimination. Elle irrigue également l'incertitude et la confusion du fondement juridique des actions,

40. CA, Versailles, 20 février 2014, n 12/04858; CA, Versailles, 26 juin 2014, n 12/02318 (série de dix décisions). 41. CA, Douai, 3 février 2012, n 11/00201 (série de dix décisions) ; voir de même, Soc., Orléans, $n^{\circ}$ 11/01067 (six affaires).

42. Toulouse, 18 octobre $2013, \mathrm{n}^{\circ} 11 / 06117$ (série de trois décisions). 
mêlant fréquemment les règles anti-discriminatoires à d'autres fondements tels que le harcèlement moral, contribuant ainsi à brouiller la fonction de ces règles.

\section{L'ambiguïté dans la construction du raisonnement : de la méthode des panels à l'égalité de traitement}

Parmi les actions introduites sur le fondement de la discrimination syndicale, certains litiges prud'homaux sont particuliers en ce qu'ils se cristallisent sur la problématique de la preuve, et plus particulièrement de la «preuve statistique », communément appelée « panel ». Celui-ci ne constitue pas en soi un facteur d'ambiguïté des actions, mais il est souvent l'instrument d'un glissement des affaires vers des questions d'égalité de traitement. Pour Olivier LECLERC (2013, p. 83) « le recours à ce mode de preuve permet d'objectiver les effets d'une action guidée par une raison d'agir illicite, sans qu'il soit nécessaire de s'interroger sur les intentions de l'auteur de la discrimination ».

Ces «panels » sont construits à partir d'un triptyque : analyse des faits, modélisation et comparaison (SPIRE, 2006). Dans un premier temps, il s'agit de recueillir des éléments factuels relatifs à une liste de salariés recrutés à la même époque, soit avec un niveau de formation ou de qualification équivalent, soit au même coefficient. L'opération consiste à établir un groupe de comparaison pertinent et significatif. Cette étape laisse place à une deuxième qui consiste en l'élaboration d'histogrammes et de tableaux retraçant les carrières des salariés composant l'échantillon de référence. Enfin, les demandeurs à l'action comparent les déroulements de carrière des militants syndicaux aux autres, afin d'étayer l'existence d'une distorsion dans l'évolution de carrière, laquelle serait constitutive d'un élément de fait laissant supposer l'existence d'une discrimination syndicale (PÉCAUT-RIVOLIER, 2013). La méthode est admise par la Cour de cassation sans qu'elle soit figée dans ses modalités techniques (GRUMBACH, SERVERIN, 2012) $)^{43}$.

Les litiges en question s'inscrivent dans le cadre d'un régime probatoire particulier régi par l'article L. 1134-1 du Code du travail, qui organise l'administration de la preuve en deux temps. Dans un premier temps, il appartient au salarié qui s'estime victime de discrimination syndicale d'apporter des éléments de fait laissant supposer l'existence d'une discrimination. Le salarié n'a pas à apporter la preuve de la discrimination, mais il lui revient d'établir des «éléments de fait susceptibles de caractériser une atteinte au principe d'égalité de traitement ${ }^{44} \gg$. Dans un second temps, il appartient alors à l'employeur de démontrer que la mesure litigieuse ou le déroulement de carrière sont justifiés par des éléments objectifs étrangers à toute discrimination ${ }^{45}$. L'intervention du juge consiste à examiner le déroulement de carrière du salarié et les agissements de l'employeur qui seraient intervenus dans ce cadre pour vérifier les allégations de

43. Soc., 3 juillet 2012, $\mathrm{n}^{\mathrm{o}} 10-25.747$, et 24 octobre 2012, $\mathrm{n}^{\circ} 11-12.295$.

44. Soc., 28 mars 2000, pourvoi $n^{\circ} 97-45258$.

45. Voir l'article L. 1134-1 du Code du travail. 
discrimination syndicale du salarié : il appartient au juge de « vérifier, en présence d'une discrimination syndicale invoquée, les conditions dans lesquelles la carrière du salarié s'était déroulée et alors que la preuve de la discrimination n'incombait pas à celui-cir ${ }^{46} \gg$.

L'analyse du contentieux de la lutte contre la discrimination syndicale met en évidence l'ambiguïté de la construction de certaines actions présentées sur le fondement de la discrimination syndicale et construites sur la seule base de "panels », ou de comparaisons, si bien que les débats relèvent in fine d'une logique d'égalité de traitement. Ainsi, c'est moins l'existence d'une mesure de rétorsion à l'égard d'une activité militante, collective ou mutualiste qui est en cause, que la revendication d'une évolution de carrière moyenne en comparaison d'autres salariés.

Cette configuration litigieuse est illustrée par deux arrêts rendus par la cour d'appel de Pau le 4 septembre $2012^{47}$. Deux salariés ayant trente-quatre et trentesix ans d'ancienneté ont saisi la juridiction prud'homale afin de faire condamner leur employeur pour discrimination syndicale dans l'évolution de carrière. Leur syndicat s'est également joint à l'instance, en qualité d'intervenant volontaire, pour obtenir réparation du préjudice qui lui était causé en tant qu'organisation habilitée à défendre un intérêt collectif (COHEN, 1990).

Le débat s'engage alors sur la pertinence du « panel », notamment sur l'ancienneté et les coefficients de quatre salariés de l'entreprise retenus pour l'établissement d'un périmètre de comparaison. Il s'agit ici d'apprécier une éventuelle distorsion dans l'évolution de carrière : «l'examen des courbes de progression des carrières montre que celle de Monsieur Guy X est la plus faible des quatre salariés du panel sur l'ensemble de la carrière et si on les examine comparativement à compter de la date des dernières embauches du panel en 1986, la courbe d'évolution de carrière de Monsieur Guy X est quasiment plate à côté de celle de ses collègues ». En définitive, la cour retient que, compte tenu de l'absence d'explication objective et pertinente de la disparité de rémunération, le salarié est bien victime de discrimination syndicale.

Un autre exemple illustre la nouvelle tournure prise par les actions en justice sur le fondement de la discrimination syndicale. Il est tiré d'une série d'arrêts rendus par la cour d'appel de Paris le 29 novembre 2012, contre la société Sagem ${ }^{48}$. La demande prend appui sur un panel réalisé par un inspecteur du travail, qui compare la situation des titulaires de mandats syndicaux avec celle des salariés exerçant le même métier et embauchés à la même période. L'absence de réponse de l'employeur face à cette inégalité de traitement, sans autre référence à l'activité militante des salariés, constitue un élément de fait laissant supposer l'existence d'une discrimination face à laquelle l'employeur ne parvient pas à démontrer l'existence d'éléments objectifs étrangers à toute discrimination.

46. Soc., 27 novembre 2002, $\mathrm{n}^{\circ} 00-44736$ (inédit).

47. CA, Pau, 4 septembre 2012, $\mathrm{n}^{\circ}$ 10/04765 et 10/04767.

48. CA, Paris, 29 novembre 2012, ${ }^{\circ}$ 10/06462, 10/06463, 10/06464 et 10/06465. 
L'utilisation de «panels » ou de comparaisons n'est pas en soi ambiguë dès lors qu'elle constitue un moyen parmi d'autres d'établir des faits laissant supposer l'existence d'une discrimination syndicale, ou de chiffrer le préjudice subi par les salariés. Toutefois, l'observation des contentieux montre assez clairement que leur mobilisation devant les juridictions conduit à un dévoiement des règles prohibant les discriminations. En effet, les débats conduits par les parties se concentrent exclusivement sur des comparaisons, comme s'il s'agissait de mettre en œuvre une règle d'égalité de traitement.

Sur le plan jurisprudentiel et doctrinal, le fondement juridique de la discrimination se distingue de l'égalité, ainsi que l'illustre un arrêt en date du 27 octobre $1999^{49}$ au travers duquel la Cour de cassation énonce « qu'une différence de traitement entre les salariés d'une même entreprise ne constitue pas une discrimination illicite au sens de l'article L. 122-45 du Code du travail ». Suivant cette perspective, il a été précisé que les deux principes ne relèvent pas de la même logique : dans le cadre de la discrimination, il s'agit de prohiber le recours à un critère de discrimination particulier et, le cas échéant, de contrôler la validité d'un tel recours ; pour l'égalité de traitement, il est davantage question de s'assurer que « l'octroi d'un avantage obéisse à des critères objectifs, pertinents, constants, vérifiables, qu'il s'agisse d'évaluer l'identité ou la comparabilité des "situations" des intéressés, ou qu'il s'agisse d'apprécier la légitimité de motifs d'une éventuelle différenciation entre salariés placés dans des situations comparables » (JEAMmaUd, 2004 ; PORTA, 2011a et b).

Ces considérations, qui structurent la distinction entre les principes d'égalité et de non-discrimination, permettent, en outre, de singulariser la problématique de la discrimination syndicale, au regard des autres formes de discrimination.

En effet, la lutte contre la discrimination syndicale a pour terrain d'élection le combat contre la répression syndicale perpétrée par l'employeur et ayant vocation à nier la liberté individuelle et collective des salariés à se constituer en un collectif de travail susceptible de porter des revendications. Cette lutte ne s'inscrit pas seulement comme la défense de principes ou la sanction de mesures illicites, mais peut être tournée aussi vers la conservation d'une capacité d'action et de mobilisation. Pourtant, lorsqu'elle s'appuie exclusivement sur des comparaisons de «panels », la revendication d'une capacité d'action se transforme en une revendication d'évolutions de carrières moyennes pour les salariés titulaires de mandats ou syndiqués. Elle se traduit, de fait, le plus souvent par l'obtention d'une « pension » allouée à la suite d'un procès engagé en fin de carrière. En outre, cet usage particulier des «panels » dans la lutte judiciaire contre les discriminations syndicales est potentiellement ambivalent, dès lors que les entreprises ou groupements patronaux s'engagent dans la production de contre-panels, multipliant les variables de comparaison et les instruments statistiques afin de brouiller les frontières des groupes de salariés qui sont l'objet des comparaisons. 
À cet égard, le raisonnement tenu par l'employeur à l'occasion de deux arrêts rendus par la cour d'appel de Versailles le 29 janvier $2013^{50}$ est assez significatif de la réversibilité de l'usage de ces panels (LyON-CAEN, 1995). Dans l'un d'eux, un salarié est embauché en 1980 chez Renault en qualité d'ouvrier. Il connaît une progression régulière de son coefficient conventionnel, mais celle-ci s'interrompt brusquement à partir de 1995, date à laquelle le salarié prend son premier mandat de représentant du personnel. Il saisit la juridiction prud'homale, afin de voir reconnaitre la discrimination dont il s'estime victime. Le salarié fait état d'une pluralité d'éléments laissant supposer l'existence d'une discrimination, notamment la mention de son activité syndicale dans plusieurs comptes rendus d'évaluation, ainsi qu'un panel retraçant son évolution de carrière comparativement à celles d'autres salariés placés dans des circonstances analogues. À eux seuls, ces éléments permettent de présumer de l'existence d'une discrimination syndicale, d'autant que l'employeur n'est pas, au regard des mentions litigieuses, en mesure d'apporter des faits démontrant le contraire. En outre, la société Renault reconnaît que l'entretien individuel d'évaluation est déterminant pour les changements de coefficients : un lien de causalité direct entre l'évaluation défavorable et l'absence d'évolution de carrière peut ainsi être établi. La cour retient que le salarié a apporté des éléments de fait laissant supposer l'existence d'une discrimination tandis que l'employeur n'apporte aucun élément objectif susceptible de démontrer le contraire.

Le salarié ne produit un panel de comparaison que dans un second temps, afin d'évaluer le préjudice subi. Il y regroupe des salariés ayant sensiblement le même âge, la même ancienneté que lui, entrés chez Renault à la même période, avec une formation professionnelle comparable et des niveaux de qualifications et de compétences analogues aux siens au début de la période litigieuse. Affirmant que la comparaison devait être effectuée avec des salariés actuellement placés dans les mêmes conditions, l'employeur produit alors un contre-panel prétendant que la comparaison devait être effectuée au regard des salariés actuellement placés dans les mêmes conditions, ce qui lui permettait subrepticement, d'évincer du périmètre de référence, les salariés promus cadres.

Sur ce point, la cour répond que l'employeur ne fournit pas d'éléments tels que le suivi de formations qualifiantes, qui seraient de nature à justifier l'évolution de ces salariés vers le statut de cadre. Elle valide ainsi le « panel » de comparaison établi par le demandeur et condamne l'employeur pour discrimination syndicale. L'argumentation ici développée par l'employeur est intéressante et illustre le caractère possiblement ambivalent de l'usage par l'employeur des «panels ». Ce dernier procède à un maniement habile des divers périmètres de comparaison afin de déplacer l'analyse de la situation des salariés non pas au moment du recrutement mais de l'action en justice. Le salarié obtient finalement gain de cause, en raison d'une construction solide de l'argumentation juridique étayée par des éléments matériels tels que les entretiens 
d'évaluation et non sur le terrain de l'égalité de traitement, comme l'y invitait justement l'employeur.

L'ambiguité dans la construction du contentieux de la discrimination syndicale peut également être liée à la multiplication des fondements juridiques.

\section{L'ambiguïté dans la combinaison de la discrimination syndicale et du harcèlement moral}

D'autres actions présentent une certaine confusion en ce qu'elles emportent dans un même mouvement discrimination syndicale et harcèlement moral, alors que l'un comme l'autre relèvent de logiques différentes.

Le harcèlement moral, défini aux articles L. 1152-1 du Code du travail et 222-33-2 du Code pénal, vise à protéger la personne du salarié, dans son intégrité physique et mentale, contre les agissements de l'employeur, d'un supérieur, voire d'un collègue (COEURET, ForTis, 2012). Cette finalité paraît éloignée de celle reconnue à la prohibition des discriminations syndicales, qui, comme nous l'avons vu, ne tend pas seulement vers la protection de la personne, mais aussi vers la protection de l'exercice d'une liberté et d'une capacité d'action individuelle et collective portée par l'intérêt de la collectivité de travail. Ces distinctions élémentaires n'empêchent pas les demandeurs de mobiliser conjointement les deux fondements juridiques dans le cadre d'un même litige et d'une même argumentation.

Cette confusion, qui fait perdre à la discrimination syndicale toute dimension collective et militante, se retrouve dans plusieurs décisions de l'échantillon étudié. Parmi notre corpus de 124 décisions en appel des juridictions civiles recensées au dernier trimestre 2014 (cf. supra, tableau 2), 11 arrêts sont concernés ${ }^{51}$, dont une série de six arrêts dans une même entreprise.

La décision rendue par la cour d'appel de Caen le 19 décembre 2014 (précitée) illustre cet usage du fondement de la discrimination syndicale devant les juges. En l'espèce, un salarié titulaire d'un mandat syndical CFDT depuis 2006 agit en justice afin d'obtenir la résiliation de son contrat de travail et diverses sommes au titre de son exécution. Mais ce qui retient plus particulièrement l'attention est la demande de dommages et intérêts formulée sur le fondement de « discrimination syndicale et harcèlement moral ».

Dans ce cadre, plusieurs griefs sont reprochés à l'employeur. Ce dernier est accusé d'avoir proféré des menaces de sanctions disciplinaires à l'encontre du salarié, d'avoir été à l'origine de « tracasseries »- lesquelles résulteraient de la conduite d'un camion potentiellement dangereux vers un garage alors que le salarié n'était pas le chauffeur habituel du véhicule - et d'avoir perpétré des mesures vexatoires dans le cadre d'un

51. CA, Caen, 19 décembre 2014, n 12/01784; CA, Angers, 25 novembre 2014, n 12/02414; CA, Nancy, 17 octobre 2014, n $^{\circ}$ 12/01373 ; CA, Nancy, 3 octobre 2014, n ${ }^{\circ}$ 13/01287 ; CA, Paris, 11 décembre 2014, n 11/08306 ; CA, Paris, 29 novembre 2014, n 12/07579; CA, Paris, 19 novembre 2014, n $12 / 03704$. 
refus de prise de congés. La cour rejette l'ensemble de ces éléments, comme ne laissant présumer ni l'existence d'un harcèlement moral, ni d'une discrimination syndicale. Toutefois, l'argumentation du salarié sera retenue pour ce qui est de la tentative de licenciement infructueuse : un refus de l'inspection du travail conjugué à une agression physique sera pris en compte par le juge, comme laissant supposer l'existence d'une situation de harcèlement moral.

Notons qu' au fur et à mesure de l'argumentation, le fondement juridique de la discrimination syndicale disparaît dans l'analyse des faits pour ne laisser place qu'à une démonstration partielle du harcèlement moral. Ainsi, il apparaît que la discrimination constitue le point de départ du raisonnement. Pour les demandeurs, c'est l'exercice du mandat qui constitue le motif originel des mesures de rétorsions subies par le salarié. Toutefois, il n'est ensuite jamais fait référence à un quelconque exercice du mandat, le terme de discrimination syndicale s'estompant pour laisser place à la démonstration d'un harcèlement moral, sans que les caractéristiques propres au harcèlement ne soient mises en avant. In fine, l'employeur est condamné pour « harcèlement moral » alors que la demande initiale portait sur la « discrimination syndicale et harcèlement moral », sans que le salarié soit débouté pour la demande tenant à la discrimination syndicale. Le fait que le terme ait disparu de l'argumentation reflète bien le caractère évanescent des actions menées sur ce fondement dans ce type d'affaire.

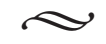

L'analyse que nous avons conduite comporte des limites, qui invitent à rester prudent quant aux enseignements que l'on peut en retirer. On se gardera d'abord de déduire des observations de type quantitatif des conclusions générales sur l'état de la réglementation ou la manière dont elle influe, en dehors des contentieux observés, sur les comportements des entreprises ou des militants syndicaux. Il serait par exemple hâtif de conclure, de l'abondance ou de la rareté des actions en justice, des indications sur le respect ou la méconnaissance des règles relatives aux discriminations dans l'entreprise. Des contentieux peu abondants peuvent aussi bien résulter d'un respect des règles par les acteurs du fait de l'efficacité des dispositifs mis en place - ce que suggère le faible volume contentieux sur les réintégrations au mépris d'un refus d'autorisation de licencier - que de la difficulté éprouvée pour mobiliser un certain nombre de règles : cela semble être le cas pour les sanctions pénales des discriminations syndicales. L'analyse quantitative des décisions doit donc simplement prendre acte de la variété des litiges et de la pluralité des stratégies de mobilisation de ces règles (SERVERIN, 2014).

Toutefois, l'analyse systématique des décisions de justice est intéressante à double titre : elle permet de comprendre les objets et les enjeux des litiges. D'un point de vue qualitatif, l'observation révèle la part spécifique prise par les contentieux sur les discriminations syndicales, se distinguant ainsi de ceux qui n'exigent pas la mobilisation de ce corps de règles. Il apparaît à ce titre que les règles anti-discriminatoires sont 
essentiellement orientées vers une protection individuelle des droits qui peut parfois venir en complément des règles orientées vers la protection de l'exercice des prérogatives de représentation des intérêts du personnel, lorsque celui-ci est invoqué : la protection du droit à l'égalité de rémunération due aux représentants du personnel, grâce à la production de panels de comparaison, en présente un parfait exemple. L'étude des contentieux montre que la mobilisation de ces normes parait le plus souvent orientée vers une finalité beaucoup plus individuelle. Elle peut ainsi devenir un moyen de revendiquer un droit individuel à une carrière " moyenne », quand ce n'est pas un droit à réparation pour des faits difficiles à caractériser, à la frontière entre la discrimination et le harcèlement. Il apparaît que ces normes tendent à perdre de leur spécificité dans la déclinaison qui en est faite devant les juridictions, se confondant souvent avec les normes d'égalité de traitement d'une part et l'interdiction du harcèlement moral d'autre part. Le motif syndical ne figure plus alors qu'en arrière-plan, sans que les juridictions parviennent véritablement à opérer un lien avec l'exercice des fonctions représentatives ou de l'activité militante.

Une telle mobilisation conduit à s'interroger sur les raisons d'être d'un corps de règles spécifiquement destinées à appréhender les discriminations syndicales. S'il présente une utilité qui ne peut être déniée lorsqu'il vient en appui de l'exercice des prérogatives de représentation des intérêts des salariés, il ne saurait en être totalement détaché, au risque de se prêter à une instrumentalisation individuelle de ces droits, qui risque de décrédibiliser le sens même de l'action syndicale.

\section{BIBLIOGRAPHIE}

Bossu B. (dir.) (2014), Les Discriminations dans les relations de travail devant les cours d'appel. La réalisation contentieuse d'un droitfondamental, Recherche réalisée avec le soutien de la Mission de recherche Droit et Justice, rapport, Lille, Centre de recherche Droit et perspectives du droit.

ChaPpe V.-A. (2013), « Dénoncer en justice les discriminations syndicales : contribution à une sociologie des appuis conventionnels de l'action judiciaire », Sociologie du travail, vol. 55, $\mathrm{n}^{\circ} 3$, pp. 302-321.

ChapPe V.-A. (2013), L'Égalité en procès. Sociologie politique du recours au droit contre les discriminations au travail, thèse de doctorat en sciences sociales, sociologie, École nationale supérieure de Cachan.

COEURET A., ForTis É. (2012), Droit pénal du travail. Infractions, responsabilités, procédure pénale en droit du travail et de la sécurité sociale, Paris, Lexis Nexis, $5^{\mathrm{e}}$ édition.

Cohen M. (1990), « Le droit de substitution, cadeau empoisonné aux syndicats », Droit social, $\mathrm{n}^{\circ} 11$, pp. 790-793.

Grumbach T., Serverin É. (2007), «Une prestation de "coaching" antisyndical devant les juridictions pénales », Revue de droit du travail, $\mathrm{n}^{\circ} 5, \mathrm{pp} .332-335$. 
Grumbach T., Serverin É. (2012), « Discrimination syndicale : le concours des panels ne doit pas être arbitré par la Cour de cassation. Soc. 3 juillet 2012, n ${ }^{\circ}$ 10-25.747, inédit ; Soc. 24 octobre 2012, $\mathrm{n}^{\circ}$ 11-12.295, inédit », Droit social, $\mathrm{n}^{\circ} 12$, pp. 714-718.

Guilloneau M., SERVERIN É (2015), « Les litiges individuels du travail de 2004 à 2013 : des actions moins nombreuses mais toujours plus contentieuses », Infostat justice, $\mathrm{n}^{\circ} 135$.

Guiomard F. (2013), « Constituer une typologie des actions en justice en matière d'égalité et de discrimination ? », in Borenfreund G., Vacarie I. (dir.), Le Droit social, l'égalité et les discriminations, Paris, Dalloz, pp. 61-76.

Guiomard F., Meftah I. (2013), « Usages et mésusages de la discrimination syndicale », in Serverin. É., Guiomard F. (dir.), Des revendications des salariés en matière de discrimination et d'égalité. Les enseignements d'un échantillon d'arrêts extrait de la base JURICA (2007-2010), Rapport pour la Mission de recherche Droit et Justice, pp. 151-159.

Jeammaud A. (2004), « Du principe d'égalité de traitement des salariés », Droit social, $\mathrm{n}^{\circ}$ 7-8, pp. 694-702.

LANQuetin M.-T. (2010), « V Discrimination », Répertoire de droit du travail Dalloz, Paris, Dalloz.

LECLERC O. (2013), «Égalité des personnes et modes de preuve. À propos des usages du raisonnement statistique dans la preuve des discriminations », in Borenfreund G., Vacarie I. (dir.), Le Droit social, l'égalité et les discriminations, Paris, Dalloz, pp. 77-94.

LyOn-CAEn G. (1995), Le Droit du travail. Une technique réversible, Paris, Dalloz.

Munoz Perez B., Serverin É. (2005), Le Droit du travail en perspective contentieuse, Paris, ministère de la Justice.

OBSERVATOIRE DE LA DISCRIMINATION ET DE LA RÉPRESSION SYNDICALES (2014), De la discrimination individuelle à l'action collective : propositions pour garantir le respect des droits syndicaux et salariés, Rapport 2014.

PAK M., PignONi M.-T. (2014), « Les représentants du personnel : quelles ressources pour quelles actions ? », Dares Analyses, $n^{\circ} 84$.

Pignoni M.-T. (2016), « La syndicalisation en France. Des salariés deux fois plus syndiqués dans la fonction publique », Dares Analyses, $\mathrm{n}^{\circ} 25$.

PÉCAUT-RIVOLIER L. (2013), Rapport sur les discriminations collectives en entreprise. Lutter contre les discriminations au travail : un défi collectif, Rapport pour les ministères du Travail, de la Justice et des Droits des femmes.

Petit F. (2004), « L'action de substitution, un cadeau promis à un avenir meilleur », Droit social, $\mathrm{n}^{\mathrm{o}} 3$, pp. 262-271.

PorTa J. (2011a), «Discrimination, égalité et égalité de traitement. À propos des sens de l'égalité dans le droit de la non-discrimination ( $1^{\mathrm{re}}$ partie) », Revue de droit du travail, $\mathrm{n}^{\mathrm{o}} 5$, pp. 290-297.

PORTA J. (2011b), «Égalité, discrimination, égalité de traitement. À propos des sens de l'égalité dans le droit de l'égalisation ( $2^{\mathrm{e}}$ partie) », Revue de droit du travail, n ${ }^{\circ} 6$, pp. 354-362. 
Entre égalité de traitement et harcèlement, quel fondement juridique de la discrimination syndicale ?

SERVERIN É. (2009), «Plaidoyer pour l'exhaustivité des bases de données des décisions du fond (à propos de l'ouverture à la recherche de la base JURICA) », Recueil Dalloz, n ${ }^{\circ} 43$, pp. 2882-2887.

SERVERIN É., GUIOMARD F. (dir.) (2013), Des Revendications des salariés en matière de discrimination et d'égalité. Les enseignements d'un échantillon d'arrêts extrait de la base JURICA (2007-2010), Rapport pour la Mission de recherche Droit et Justice.

SERVERIN É. (2014), « Introduction. Une enquête juridique sur la place du collectif dans la justice contemporaine », in Omarjee I., Sinoppoli L. (dir.), Les Actions en justice au-delà de l'intérêt personnel, Paris, Dalloz, p. 3.

SPIRE R. (2006), «Agir contre la discrimination syndicale au travail : le droit en pratique », Le Droit ouvrier, nouvelle série, $\mathrm{n}^{\circ}$ 693, pp. 170-218 et 222-230.

VERDIER J.-M. (1984), Droit du travail. 5, Syndicats et droit syndical. 2, Le droit syndical dans l'entreprise, $2^{\mathrm{e}}$ édition, Paris, Dalloz.

Verdier J.-M. (1987), Droit du travail. 5, Syndicats et droit syndical. 1, Liberté, structures, action, $2^{\mathrm{e}}$ édition, Paris, Dalloz. 